\title{
Fog Enabled Cloud Based Heart Rate Monitoring System
}

\author{
Arulmozhiselvan $\mathrm{L}^{\mathrm{a}, 1}$ and $\mathrm{Uma}^{\mathrm{a}}$ \\ a Department of Information Science and Technology, Anna University, chennai, India
}

\begin{abstract}
The world has seen a substantial growth in the number of senior citizens who often endure from heart disease in recent times and has seen a major spread of the viral disease (COVID-19) in recent months, resulting in thousands of deaths, particularly among the elderly and people suffering from heart disease. Among the most promising health care that seek to relieve the suffering of patients, particularly the elderly, by eliminating the trouble of going to hospital centers for treatment and enabling them to obtain medical attention in their homes. The IoT has been depended on and its use has seen broad adoption in health care, where it is commonly used remotely to track patient health. Fog computing expands the computational capabilities of the cloud to the edge devices of the IoT, enabling many smart devices in healthcare to provide services such as storing and retrieving information to their users. However in the traditional cloud-based system, there is a timing delay in providing a reliable and secure heart monitoring system. The main objective of this work is to develop a fog-enabled cloud-based (FECB) heart rate monitoring unit that allows better network use and energy consumption. In terms of performance metrics, such as latency and delay, the proposed system also outperforms the existing system.
\end{abstract}

Keywords. Cloud Computing, Fog Computing, Healthcare, IoT, Patient monitoring.

\section{Introduction}

Medical care now plays an important role in people's personal well-being, with healthcare professionals, distributors, patients, and corporations massively pushing and adopting these services. Patients have to be taken to a hospital or health centers in the conventional healthcare setup to monitor and track their health issues locally. Healthcare services have recently been implemented with cloud computing services on data centers with front-end smart healthcare to regulate, analyze and visualize data captured in real-time from IoT devices. Remote patient health surveillance helps to reduce hospitalizations by 50 per cent, emergency room visits by 73 per cent, and patient costs by 51 per cent. In order to draw certain assumptions that are useful in maintaining a healthier lifestyle, there are many health applications such as Microsoft Health, Apple Health Kit, Samsung S Health and Google Fit to gather patient health data by tracking their everyday activities (e.g. sleeping practices, exercise routines and food choices) [1]. As patients are

\footnotetext{
${ }^{1}$ L Arulmozhiselvan, Department of Information Science and Technology, Anna University, chennai.

E-mail: arulmozhiselvan@auist.net
} 
forced to stay in the hospital for the duration of their treatment due to the lack of technology capable of delivering patient health information remotely to approved health practitioners, healthcare facilities are becoming more and more expensive. Remote patient monitoring provides tremendous opportunities to reduce the cost of patient health care and to improve the quality of healthcare services by detecting and avoiding illnesses. By allowing remote patient monitoring in hospitals and even at home, IoT will bring tremendous opportunities to revolutionize healthcare in the near future. IoT systems directly gather health information from patients and send it to healthcare professionals in real time. This would not only reduce the expense of healthcare services for patients, but also encourage health conditions to be dealt with before they become serious [2].

By the year 2020, the present hospital-centered healthcare practice is projected to be balanced by its home base equivalent in the next decade. The average growth in health data will also be around 48 percent a year and the amount of health data will grow to 1018 bytes by 2020. It takes some time to allocate vast volumes of data to cloud computing for processing and storage, which is inappropriate in life or death circumstances. Architecture with a middle computing layer is becoming the more commonly used technology to improve the quality of healthcare services to support this data development because of the growing sensing devices used for healthcare. This intermediary computing layer is known as fog computing or Edge computing, which offers unique IoT device services to improve the processing chance to react to patient requests from e-Health customers. E-Health information must be applicable in real time to medical professionals by adding this second tier between IoT body-sensor sensors and cloud computing [3].

In support of IoT-enabled clinical services offering solutions for scalability, data processing and consistency, cloud computing services have been broadly approved. The geographical centralization of cloud data centers, however, allows data obtained from sensors to be distributed for processing over a multi-hop range, adversely affecting the latency tolerance of the solutions. In addition, cloud resource management in heterogeneous healthcare environments requires complex strategy implementation in order to avoid constant revision of the distribution of resources in response to unequal and unpredictable loads of data from healthcare solutions [4-6]. By investigating lightweight and customizable additional computing tools closer to the IoT database in healthcare solutions, Fog computing is a promising option in this scenario. Conventional edge computing devices such as switches, routers, low-profile computing devices, etc. are fitted with computer infrastructure, utilities and management models for local lean technologies to be applied in this approach. As a consequence, some data processing can be carried out closer to the data source, resource demands can be dispersed, the need for multi-hop data exchange can be minimized, latency reduced and service flexibility encouraged [7]. Providing an easy sensor-to-cloud architecture is not feasible for many healthcare applications especially because most hospitals will not prefer outside storage of patient data. There is also often the grim case of a packet loss or a failure of the data centre which puts the health of patients at risk. Fog computing helps healthcare solutions in this field. Fog computing is a decentralized computer architecture that processes and stores information between the source of origin and the resources of the cloud. This contributes to the minimization of overhead data transfer and ultimately increases computational efficiency on cloud networks by reducing the need to process and store large quantities of illumination [8]. 
The Fog computing framework is largely driven by a massive rise in Internet of Things (IoT) devices, where an ever growing amount of data is created from an already array of devices (in terms of volume, variety, and velocity). The Fog model aims to provide problems with a flexible, decentralised approach. This is accomplished through the development, as shown in Figure 1, of a new hierarchically dispersed and local network between the Cloud system and end-user devices. This platform is capable of sorting, consolidating, sorting, analyzing and sharing information and will save time and energy for interaction [9]. Although the resources and computing resources of fog resources are limited, they are versatile enough to adjust according to the context of performance. The challenge of managing and running distributed computing scenarios is addressed by the convergence of variable requirements and restricted computing resources, ensuring performance, reliability and protection. During the transition of data from the sensors to the cloud and the cloud to hospitals or personal clinicians, the use of only the cloud can cause delays. In healthcare, we have emergency service systems that involve real-time operations that play an important role in productivity and time; this can suffer as a result of cloud delays. Therefore, not only because of latency problems, but also because of protection, the transfer of such enormous quantities of data back and forth is not an effective option. The risks associated here are not only data breaches, but also risks to patients' health [10]. Therefore, in this paper a fog-enabled cloud-based (FECB) heart rate monitoring unit that allows better network use and energy consumption. This approach also reduces latency. Along with this detailed study of cloud and fog computing contribution in Section 2 provides the related work. Section 3 describes the proposed health

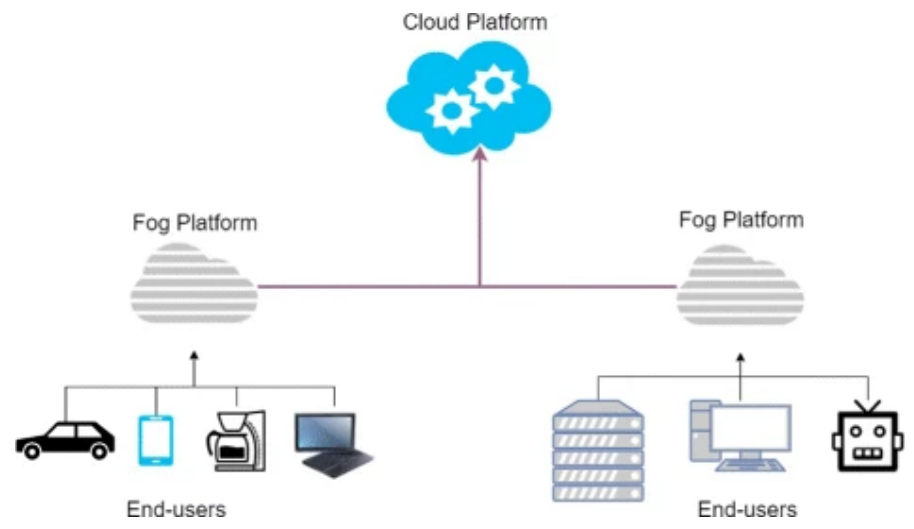

Figure 1. Fog enabled cloud platform healthcare

care monitoring unit and results and discussions are provided in Section 4 followed by conclusion in Section 5.

\section{Background Study}

Fog computing enables organizations, especially in large environments where devices need to communicate with each other and the data centre, to use cloud computing to support IoT [11]. This makes it possible for devices that access similar data to communicate through a sub-cloud network called a fog layer. Fog computing enhances opera- 
tional performance by providing end users to access smaller, more specific information rather than obtaining information from users in a centralised, cloud-based infrastructure together with information they would never need to access. Hence, in this section, fog computing in healthcare related works is discussed.

IoT-Fog-Cloud [12] presented study, models, and demonstrates how to lower the cost of computational power while maintaining performance limitations for health requestsparticularly response time of obtaining medical data stored in a fog-cloud environment. This work presented a queuing model to forecast the minimum necessary amount of computing resources (both fog and cloud nodes) to satisfy the Service Level Agreement (SLA) for responsiveness in order to address this problem. Via nonlinear models, we check and cross check our analytical model. The conclusions drawn from our analytical models indicate that in order to obtain the required responsiveness under various workload conditions, the proposed model is able to properly and effectively estimate the number of computational resources needed for health data services.

A Fog-based IoT-Healthcare technology framework [13] and the incorporation of Cloud-Fog services into the conventional Cloud-based framework of interoperable clinical services. Via simulations using the iFogSim simulator, the scenarios are tested and the outcomes analysed in relation to numerical computation, latency reduction, data transmission optimization and energy consumption. In terms of cost, network delay and energy use the empirical setup point to enhancement. Integrated IoT and Fog to deliver health data [14] presented the move from the cloud to the edge network of computing knowledge. Fog computing functions closer to the customer, on the edge of the network, allowing precise service delivery with low response time to prevent delays and network failures that may disrupt or delay the decision process and delivery of healthcare services. A design framework is designed and the advantages of the IoT and fog computing integration are demonstrated by a series of use cases.

A design that focuses on healthcare systems [15], in addition to a system implementing this architecture, an architecture comprising three layers; objects (i.e., sensors), fog nodes and a cloud data centre is proposed. This arrangement provides coordination between fog nodes with efficient resource management and job allocation, which is capable of achieving a high QoS (i.e. low latency) in a healthcare service scenario. Smart devices are not capable of handling IoT-produced data as elements of IoT-driven health systems, nor is data uploading to the Cloud the required solution.

The paradigm shift, known as Fog computing, has seemed to solve the limitations of smart devices and the cloud, where an extra element collects the message and transmits the results to the cloud. Leading proponents brought into IoT-based environments by Fog computing, privacy and security concerns remain the primary challenge for its deployment. This paper presents the reasons for the convergence of the IoT-based healthcare system and Fog computing, advantages and challenges, as well as the proposal of a simple low-cost system [16].

Healthcare companies have become more open to cloud computing over the past year as a common technology for health IT infrastructure. Firms recognize that the cloud facilitates even more intelligent collaboration between health information systems, enabling smarter interaction between mission-critical platforms for end users. The need for end-to-end visibility of the cloud environment is acknowledged by organizations. However, for the sake of improved visibility, as cloud ecosystems expand, healthcare organizations are faced with adapting to the volume of data. Fog computing enables such data 
to be separated by IT administrators and users, making it easier to access and handle. The implementation of a fog layer into the architecture of cloud computing eliminates the security risk whenever it comes to patient data not being lost due to a data centre failure. But at the same time, in the cloud, the data is processed. This raises the threat to patient information privacy and also takes huge delay in accessing the information. Health monitoring system for people who are old and disabled [15] was proposed to monitor their health regularly. Heart beat rate is monitored at regular time period and collected. The collected value is compared with prescribed doctor value whether it is normal or not. If there is critical in heart beat rate then alarm is emerged and message sent to patient doctor and family member. The message is passed which contains collected heart beat rate at period of time along with patient location.

\section{System model}

Although the number of medical devices is rising day by day, most of them use preconfigured applications based on artificial intelligence to handle medical data. This suggests however that practically all of the care and evaluation decisions are taken based on the description of the common patient. In addition, due to the fact that all data analysis takes place on a central server, as more and more medical devices are linked to the system, networks also struggle from network latency issues. To build and incorporate a monitoring system for the heart beat that would allow the medical data of the patient to be processed on a Fog node using algorithm to minimize the alert delay while not exceeding the communication overhead of the server.

\subsection{Basic components}

1. A database server which preserves and manages sensed information for the patient.

2. A smartphone to provide a 2-way data stream with the sensor and the database server.

3. A lightweight, wearable body sensor that is connected to the patient with a lot of computing power.

\subsection{Algorithm}

- Uploading sensed information.

- Storing basic information about smartphone users.

- Validating input data.

- Display the heart rate is critical or not

\subsection{Responsibility of fog node}

1. Using an encrypted key, reading heart rate from the sensor.

2. Downloading corresponding user information, sending a proper notification.

3. Sending the SMS to the caretaker when the heart rate is critical.

4. Displaying collection of recent heart rates. 


\subsection{Communication with fog nodes}

Using cloud connectivity, the contact from the database server to a fog node is finished. Cloud connectivity is an effective way for a battery to connect a database server to a computer, allowing you to send messages and updates to your device from your server. The

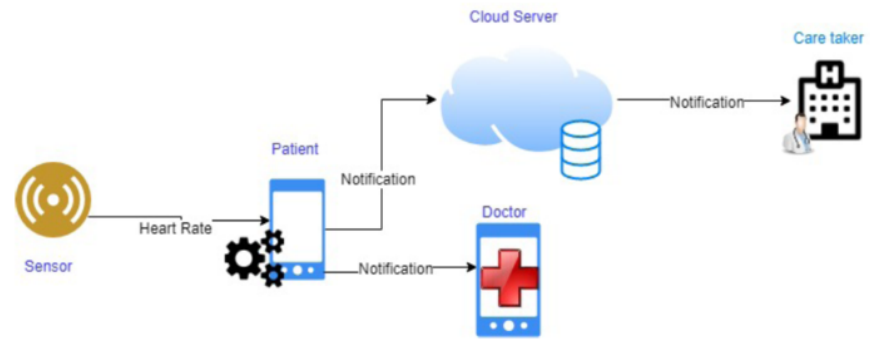

Figure 2. FECB heart rate monitoring architecture

advantage of cloud messages is that they are very simple to set up and have constructed features to handle circumstances when network issues on the side of the fog node are present. In order to send a message to the Fog node, cloud connectivity is used. The message includes information about the user that is automatically downloaded to the device after the message is sent. On the server side, there is currently no help to ensure that the message was actually received by the fog node, which allows for potential opportunities for growth.

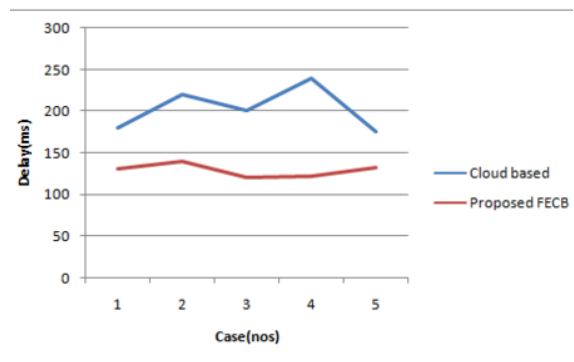

Figure 3. Comparison analysis for delay

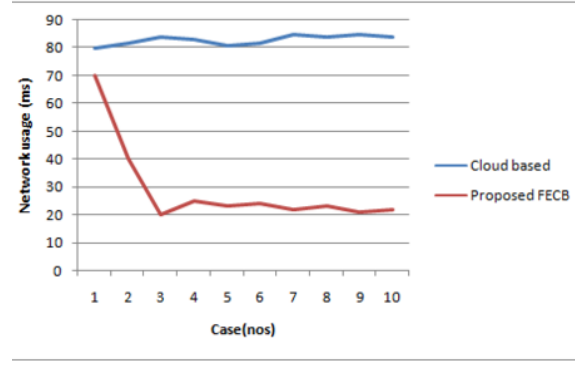

Figure 4. Comparison analysis for network usage

From Figure 2, the patient's phone transmits the heart rate to the cloud server, where the server runs the algorithm to determine whether to give the doctor a notification. If it has changed, the heart rate status is still stored in the database, so the patient's status is updated to alert the hospital caretaker. The key issue with a conventional tracking system based on cloud computing is that the extra time it takes for the data to be transmitted to the server from the patient's phone may be critical in a life and death situation. But in the proposed system the delay can be reduced using fog node installation. Figure 3 demonstrates the delay comparison analysis between conventional cloud-based heart rate monitoring and the proposed FECB monitoring unit. In this case, we consider homogeneous workloads for body sensors, which are uniformly distributed and each body 


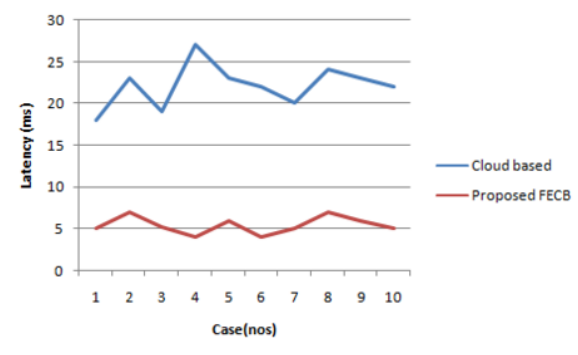

Figure 5. Comparison analysis for latency delay

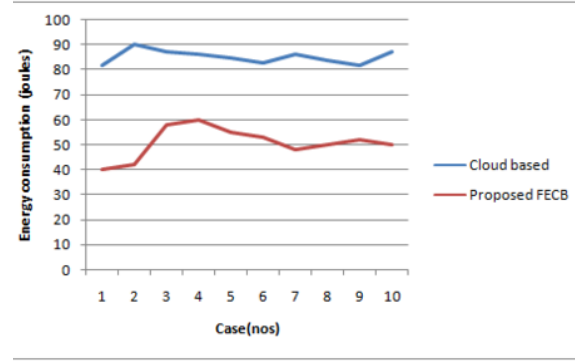

Figure 6. Comparison analysis for energy consumption

sensor generates a rate of 500 requests per second. We see the delay rises as the number of cases increases. Similarly, Figure 5 depicts the comparison analysis for latency delay and Figure 6 shows the energy consumption between traditional clouds based and proposed FECB monitoring units. We suggest that if the healthcare workload increases the response time increases with a fixed number of computing resources, and inversely, if the healthcare workload decreases the response time decreases. As a result, as a function of the forthcoming healthcare workload status, it is vital to scale up and down computing resources. In addition, the cloud computing-based approach reveals that the delayed update will reach two seconds, compared to the FECB fog-based device unit where only half of it is delayed. In addition, since this experiment was performed using a powerful high-bandwidth network signal, this delay will exponentially increasing in areas where the signal intensity or network bandwidth is smaller.

\section{Conclusion}

As a result, the FECB system for heart rate monitoring was established, providing a way for doctors and caregivers to monitor their patients. The device consists of a doctor's database server, a patient's smartphone and a sensor that supplies the application with the heart rate. In this type of environment, the use of cloud storage will only make sense if the algorithm is extremely hungry for resources and the fog node would not be capable of processing the data on a regular basis. The biggest advantage here is that all intervals are kept as short as possible as the processing happens very close to the sensor itself. In addition, since the two alerts are sent as different requests, there is a fair probability that the other one will stay untouched and the message will not be lost even though either the physician or hospital network connection breaks.

\section{References}

[1] Kraemer FA, Braten AE, Tamkittikhun N, Palma D. Fog computing in healthcare-a review and discussion. IEEE Access. 2017 May 15;5:9206-22.

[2] Malik BH, Mahmood F, Shahzad S, Arif MB, Khan WU, Ilyas S, Hassan M. From cloud computing to fog computing in Healthcare big data. InMATEC Web of Conferences 2018 (Vol. 189, p. 03011). EDP Sciences. 
[3] Priyadarshini R, Panda MR, Mishra BK. Security in healthcare applications based on fog and cloud computing. Cyber Security in Parallel and Distributed Computing: Concepts, Techniques, Applications and Case Studies. 2019 Mar 25:231-43.

[4] Shi Y, Ding G, Wang H, Roman HE, Lu S. The fog computing service for healthcare. In2015 2nd International Symposium on Future Information and Communication Technologies for Ubiquitous HealthCare (Ubi-HealthTech) 2015 May 28 (pp. 1-5). IEEE.

[5] Nandyala CS, Kim HK. From cloud to fog and IoT-based real-time U-healthcare monitoring for smart homes and hospitals. International Journal of Smart Home. 2016 Feb;10(2):187-96.

[6] Ajay H, Rao AR, Balavanan M, Lalit R. A Novel Cardiac Arrest Alerting System using IoT. International Journal of Science Technology \& Engineering. 2017;3(10):78-83.

[7] Bharati S. CLOUD AND FOG COMPUTING IN HEALTHCARE INDUSTRY 4.0. Journal of the International Academy for Case Studies. 2020 Aug 20;26(6):1-2.

[8] Abdelmoneem RM, Benslimane A, Shaaban E, Abdelhamid S, Ghoneim S. A cloud-fog based architecture for iot applications dedicated to healthcare. InICC 2019-2019 IEEE International Conference on Communications (ICC) 2019 May 20 (pp. 1-6). IEEE.

[9] Dash S, Biswas S, Banerjee D, Rahman AU. Edge and fog computing in healthcare-A review. Scalable Computing: Practice and Experience. 2019 May 2;20(2):191-206.

[10] Gill SS, Arya RC, Wander GS, Buyya R. Fog-based smart healthcare as a big data and cloud service for heart patients using IoT. InInternational Conference on Intelligent Data Communication Technologies and Internet of Things 2018 Aug 7 (pp. 1376-1383). Springer, Cham.

[11] Luo Y. Environmental cost control of coal industry based on cloud computing and machine learning. Arabian Journal of Geosciences. 2021 Jun;14(12):1-6.

[12] El Kafhali S, Salah K, Alla SB. Performance evaluation of IoT-fog-cloud deployment for healthcare services. In 2018 4th international conference on cloud computing technologies and applications (Cloudtech) 2018 Nov 26 (pp. 1-6). IEEE.

[13] Mahmud R, Koch FL, Buyya R. Cloud-fog interoperability in IoT-enabled healthcare solutions. InProceedings of the 19th international conference on distributed computing and networking 2018 Jan 4 (pp. $1-10)$.

[14] Andriopoulou F, Dagiuklas T, Orphanoudakis T. Integrating IoT and fog computing for healthcare service delivery. InComponents and services for IoT platforms 2017 (pp. 213-232). Springer, Cham.

[15] Al-Khafajiy M, Webster L, Baker T, Waraich A. Towards fog driven IoT healthcare: challenges and framework of fog computing in healthcare. InProceedings of the 2nd international conference on future networks and distributed systems 2018 Jun 26 (pp. 1-7).

[16] Maksimović M. Implementation of Fog computing in IoT-based healthcare system. Jita-Journal Of Information Technology And Aplications. 2017;14(2):100-7. 